elasticity was attached to the transducer during the elastosonography. The inlet of the carpal tunnel at the scaphoidpisiform level and the proximal portion of the carpal tunnel inlet were scanned in a transverse plane. The crosssectional area (CSA) and the elasticity of the median nerve, which was measured as the acoustic coupler/median nerve strain ratio, were evaluated. The measurements were repeated two times, and the average strain ratio was used for analysis. Results: We analyzed 342 hands in 177 RA patients (139 female, mean age: $63.5 \pm 11.6$ years) and 158 hands in 81 non-RA (68 female, mean age: $71.5 \pm 14$ years) finally. There were no significant differences in the cross-sectional area of median nerve (left: 8.9 vs $8.7 \mathrm{~mm}^{2}, \mathrm{p}=0.91$, right: 8.2 vs $8.4 \mathrm{~mm}^{2}, \mathrm{p}=0.62$ ) or the circumference of median nerve (left: $13.1 \mathrm{vs} 13.4 \mathrm{~mm}, \mathrm{p}=0.41$, right: 13.7 vs $13.7 \mathrm{~mm}, \mathrm{p}=0.95$ ) within carpal tunnel between RA group and non-RA group. Strain ratio within carpal tunnel in RA group was higher than that of non-RA group (left: 2.6 vs $2.1, p=0.002$, right: 2.7 vs $2.2, p=0.003$ ). There were no significant differences in the cross-sectional area of median nerve (left: 7.5 vs $8.1 \mathrm{~mm}^{2}$, $\mathrm{p}=0.07$, right: 8.8 vs $8.3 \mathrm{~mm}^{2}, \mathrm{p}=0.6$ ), the circumference of median nerve (left: 13.1 vs $13.5 \mathrm{~mm}, p=0.3$, right: 13.7 vs $13.9 \mathrm{~mm}, p=0.71$ ) and strain ratio (left: 2.1 vs $2.0, p=0.88$, right: 2.3 vs $2.1, p=0.01$ ) at the entrance of the carpal tunnel between RA group and non-RA group.

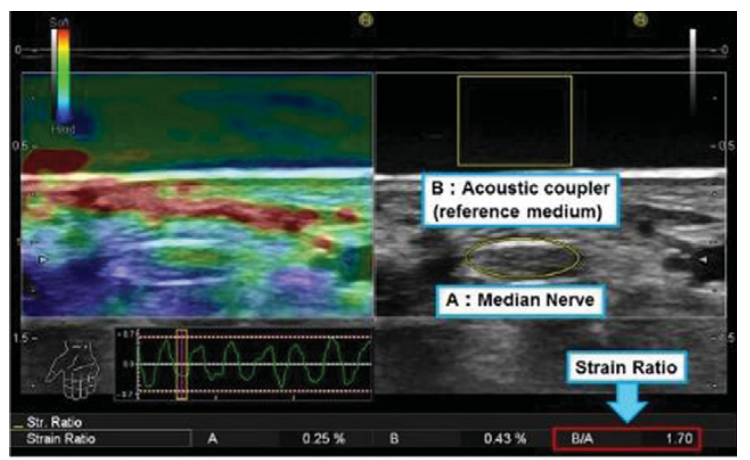

Figure: The images of acoustic coupler/median nerve strain ratio.

Conclusions: Real-time Elastosonography showed the stiffness of the median nerve with RA patients without any symptoms of CTS was higher than controls. It suggests that inflammation of flexor tenosynovitis and wrist joint may generate fibrotic change of median nerve in patients with RA.

References:

[1] Miyamoto $H$, Halpern EJ, Kastlunger M, et al. Carpal tunnel syndrome: diagnosis by means of median nerve elasticity-improved diagnostic accuracy of US with sonoelastography. Radiology. 2014;270:481-6.

Disclosure of Interest: None declared

DOI: 10.1136/annrheumdis-2017-eular.1632

\section{SAT0659 COMPARISON BETWEEN EIGHT DIFFERENT ULTRASONOGRAPHIC SCORES FOR HAND ASSESSMENT IN RHEUMATOID ARTHRITIS -A CROSS-SECTIONAL STUDY}

S. Hussain $^{1,2}$, P. Sivakumaran ${ }^{1,2}$, L. Attipoe ${ }^{2}$, C. Ciurtin ${ }^{2} .{ }^{1}$ University College London Medical School; ${ }^{2}$ Department of Rheumatology, University College London Hospitals NHS Foundation Trust, London, United Kingdom

Background: Rheumatoid arthritis (RA) is a chronic inflammatory condition associated with well-recognised inflammatory joint features, which are amenable to ultrasound (US) examination. The implementation of US scoring systems in addition to clinical examination could help standardise the way RA is monitored; however, due to variation in local availability of US and sonographer expertise, different scoring systems have been used in clinical practice (1). Despite significant research progress in supporting the role of US in RA, there is no consensus as to which scoring system is most useful.

Objectives: To assess whether simplified US protocols for hand examination correlate significantly with a 22 hand joint US score in patients with established rheumatoid arthritis, and correlate the US examination with the disease activity score (DAS-28 score).

Methods: This is a cross-sectional study of 224 RA patients stratified based on their DAS-28 scores and assessed using eight preselected US examination protocols including 22, 18, 16, 14, 10, 8 and two different combinations of 4 joints, respectively. Student T, Mann-Whitney $U$ and Kuskal-Wallis tests were employed for analysis of clinical, laboratory and US parameters in the RA patient groups $(\mathrm{P}<0.05$ was considered significant). Spearman's coefficients were used to correlate permutations of pairs of US scores, and US and DAS-28 scores.

Results: We found a significant difference between different US hand scores and their ability to detect the presence of active and chronic inflammation in RA patients. The DAS-28 scores correlated very well $(R=0.89-1, P<0.05)$ with the total Power Doppler (PD) scores generated by all US protocols irrespective of patients' disease activity. Simplified US scores missed information on presence of erosions $(\mathrm{P}<0.05)$, but were equivalent to the extensive 22 joint score in appreciating the amount of chronic and active inflammation compared to the extensive 22 joint score $(P=0.15, P=0.11$, respectively).
Conclusions: This study showed that preselected simplified US scores could be used in clinical practice to appreciate reliably the disease activity in patients with established RA; however they are less reliable in appreciating the disease burden when compared with an extended protocol for US examination of 22 hand joints. All the simplified US scores correlated very well with DAS-28 scores. References:

[1] Mandl, P., et al., A systematic literature review analysis of ultrasound joint count and scoring systems to assess synovitis in rheumatoid arthritis according to the OMERACT filter. J Rheumatol, 2011. 38(9): p. 2055-62.

Acknowledgements: S. Hussain and P. Sivakumaran contributed equally to the study.

Disclosure of Interest: None declared

DOI: 10.1136/annrheumdis-2017-eular.2722

\section{SAT0660 SAFETY, FEASIBILITY AND TOLERABILITY OF PERFORMING CONSECUTIVE MINIMAL INVASIVE ULTRASOUND-GUIDED SYNOVIAL BIOPSY PROCEDURES ON THE SAME WRIST IN A PROSPECTIVE RHEUMATOID ARTHRITIS STUDY}

S.A. Just ${ }^{1}$, C. Nielsen ${ }^{2}$, E.K. Hejbøl ${ }^{3}$, H.D. Schrøder ${ }^{3}$, I.M.J. Hansen ${ }^{4}$, T. Barington ${ }^{2}$, H.M. Lindegaard ${ }^{1} .{ }^{1}$ Rheumatology; ${ }^{2}$ Clinical Immunology; ${ }^{3}$ Clinical Pathology, Odense University Hospital, Odense; ${ }^{4}$ Medicine, Svendborg Hospital, Odense University Hospital, Svendborg, Denmark

Background: Studies on synovial tissue retrieved using the minimal invasive ultrasound-guided synovial biopsy (USG-SB) method have led to major advances in the understanding of Rheumatoid Arthritis (RA). The method is now used in multicenter RA studies and recommended in the phases of RA drug development. Only to biopsy disease active joints at start and end of a study, can lead to biopsies being retrieved from different joints. This can make interpretation of the changes in the synovial tissue and gene-expression profile difficult, as synovial histology patterns can vary between joints. We here present an approach where we biopsied the wrist with disease activity at presentation and the same wrist after six month of disease duration. We use the wrist, as it is the joint most commonly involved in the upper extremity in RA. The joint is easily accessible for USG-SB and therefore ideal to use to follow disease activity/treatment response/biomarker change in prospective RA studies.

Objectives: To assess the safety, tolerability and feasibility to perform repeated synovial biopsies from the same wrist, using a minimally invasive USG-SB technique in patients suffering from RA.

Methods: Patients with newly diagnosed untreated RA or longstanding ( $>5$ years) $\mathrm{RA}$ and at least one clinically swollen wrist, underwent $\mathrm{x}$-ray, magnetic resonance imaging (MRI) and ultrasound examination of the affected wrist and hand on the day of the biopsy. This was repeated 6 months later, where the second biopsy from the same wrist was taken. EULAR guidelines for RA treatment were followed in the 6 months between biopsies. Patient-reported outcomes (PRO) included a standard questionnaire given to all patients on the day of the biopsy as well as 2 weeks after the biopsy. Tolerability and the patient-reported willingness to repeat the procedure was assessed using the 5-point Likert scale.

Results: 38 RA patients (22 early, 16 longstanding) underwent USG-SB procedure at inclusion and after 6 months. One patient was excluded and did not have second biopsy due to diagnosis of CPPD. All patients have undergone first biopsy and at present time and $50 \%$ second biopsy. At the EULAR congress complete data will be presented. At present time, at both first and second biopsy no worsening in PRO of the biopsied joints was reported $2 \mathrm{w}$ after the biopsy, as compared before the biopsy. No infection, hemorrhage, nerve or tendon damage has currently been observed. One patient developed a tenosynovitis after biopsy (the CPPD patient), successfully treated with glucocorticoid injection. $10 \%$ of the patients were somewhat or very unlikely to have another biopsy procedure after the first procedure, and 6\% after the second. All included RA patients accepted to have a second biopsy. Detailed data on differences in tolerability between early untreated RA patients versus patients with longstanding RA, will be presented at the conference.

Conclusions: To our knowledge, we are the first, to demonstrate that retrieving synovial tissue using the USG-SB method on the same wrist, at start and end of a prospective RA study, is safe and well tolerated.

Disclosure of Interest: None declared

DOI: 10.1136/annrheumdis-2017-eular.2453

\section{SAT0661 FINGER JOINT CARTILAGE THICKNESS EVALUATED BY ULTRASOUND IN PATIENTS WITH RHEUMATOID ARTHRITIS (RA)}

T. Ogura, A. Hirata, N. Hayashi, H. Ito, S. Takenaka, Y. Fujisawa, K. Mizushina, T. Katagiri, R. Kujime, M. Imamura, N. Yamashita, H. Kameda. Toho University Ohashi Medical Center, Tokyo, Japan

Background: Joint destruction in RA includes both bone and cartilage lesions. By $X$-ray examination, cartilage destruction is evaluated as a joint space narrowing (JSN). However, joint space narrowing is not a direct evaluation of cartilage. Objectives: We aimed to examine the finger joint cartilage thickness (CT) by ultrasound (US) imaging and clarify its clinical significance in patients with RA. Methods: We enrolled 121 RA patients in low disease activity or clinical remission 
(DAS28-CRP <2.7) in this study. The CT of metacarpophalangeal (MCP) and proximal interphalangeal (PIP) joints of 2 nd to 5 th fingers was bilaterally visualized and measured at the middle portion of MCP and PIP joints from a longitudinal dorsal view, with approximately 90 degrees flexion. In addition, JSN of finger were scored by van der Heijde- modified Sharp method for patients who had a hand $\mathrm{X}$-ray within 2 months.

Results: CT in MCP joints ranged from 0.0 to $1.1 \mathrm{~mm}$ (median $0.5 \mathrm{~mm}$ ), and CT in PIP ranged from 0.0 to $0.6 \mathrm{~mm}$ (median $0.3 \mathrm{~mm}$ ), respectively. The sum of total CT from 8 fingers ranged from 4.0 to $9.4 \mathrm{~mm}$ (median $6.9 \mathrm{~mm}$ ), and there was a significant difference in total CT, but not in JSN score, between male and female patients (7.4 versus 6.7, $p=0.006$; and 11 versus $10, p=0.899$, respectively). CT was well correlated with JSN $(\rho=-0.589, p<0.001 ; \rho=-0.595$, $p<0.001$ for MCP joints and $\rho=-0.448, p<0.001$ for PIP joints), and both CT and JSN were significantly correlated with disease duration $(\rho=-0.282, p=0.002$, and $\rho=0.286, p=0.005$, respectively). Notably, CT of MCP, but not of PIP, was inversely correlated with disease duration $(\rho=-0.328, p<0.001)$. Height was correlated with CT $(\rho=0.244, p=0.008)$, but not with JSN $(\rho=-0.057, p=0.589)$. CT and JSN were not correlated with age, functional disability, and seropositivity. Conclusions: A direct visualization and quantification of finger joint $\mathrm{CT}$, especially MCP joints, by US is valid and useful in RA.

Disclosure of Interest: None declared

DOI: 10.1136/annrheumdis-2017-eular.4703

\section{SAT0662 EVALUATION OF ANTI-DOUBLE STRANDED DNA ANTIBODIES IN THE MONITORING OF SYSTEMIC LUPUS ERYTHEMATOSUS}

$\underline{\text { T. Dervieux }}^{1}$, T. O'Malley ${ }^{1}$, J. Conklin ${ }^{1}$, C. Ibarra ${ }^{1}$, C. Bentow ${ }^{2}$, M.A. Aure ${ }^{2}$ M. Mahler ${ }^{2} .{ }^{1}$ Exagen Diagnostics, Vista, $C A ;{ }^{2}$ Research and Development, Inova Diagnostics, San Diego, CA, United States

Background: Systemic lupus erythematosus (SLE) is a chronic autoimmune rheumatic disease characterized by the production of pathogenic autoantibodies. Amongst these antibodies, those directed to dsDNA are routinely used to monitor disease activity and are components of the SELENA-SLEDAI index to score severity of SLE disease. Because the predictive value of anti-dsDNA is dependent on the sensitivity and robustness of the assays used, the choice of anti-dsDNA is crucial in the clinical laboratory and clinical research setting.

Objectives: The objective was to compare four anti-dsDNA assays for their performance characteristics of SLE disease activity.

Methods: A cohort of 36 subjects with active SLE presenting with classical complement activation were enrolled and followed monthly for 1 year. At each study visit blood was collected, serum isolated and frozen until analysis. A total of 371 specimens were collected. Disease activity was scored on the day of each study visit according to the SELENA-SLEDAl method excluding anti-dsDNA or complement components (non-serological [ns] SELENA-SLEDAI). All specimens were tested using four different anti-dsDNA kits; QUANTA Lite, QUANTA Flash, a high Avidity anti-dsDNA ELISA, and the Crithidia luciliae indirect immunofluorescence assay (CLIFT) (Inova Diagnostics, San Diego, CA). Study visits presenting with inactive disease (ns-SELENA- SLEDAI score $=0$ ) were compared to those presenting with active disease (ns-SELENA- SLEDAl>0). The longitudinal data were analyzed using linear mixed effect modeling with the ns-SELENA-SLEDAI as dependent variable and the anti-dsDNA titers as fixed effect predictors. Marginal $\mathrm{R}^{2}$ was calculated for each assay.

Results: At enrollment the sensitivity of the QUANTA Lite and High Avidity anti-dsDNA both reached $64 \%$; whereas anti-dsDNA positivity was $83 \%$ by QUANTA Flash and reached $96 \%$ by CLIFT. Study visits with active disease presented with several fold higher anti-dsDNA titers than those with inactive disease status (Table 1). Linear mixed effect modeling indicated that the decrease in ns-SELENA-SLEDAI scores were associated with significant reduction in titers of all three anti-dsDNA kits (Table 2). QUANTA Flash yielded highest marginal R $^{2}$ (0.112) (Table 2).

Table 1. Anti-dsDNA antibody and disease activity

\begin{tabular}{lcccc}
\hline Anti-dsDNA & & Inactive Disease & Active disease & $p$ value \\
& & $0(\mathrm{n}=141)$ & $1+(\mathrm{n}=230)$ & \\
\hline QUANTA Flash & Titers (units) & $72(30-134)$ & $170(56-813)$ & $<0.001$ \\
& Percent positive $(>35$ Units $)$ & $72 \%$ & $86 \%$ & $<0.001$ \\
High Avidity & Titers (units) & $36(12-170)$ & $129(47-775)$ & $<0.001$ \\
& Percent positive $(>30$ Units) & $56 \%$ & $77 \%$ & $<0.001$ \\
QUANTA Lite & Titers (units) & $343(106-624)$ & $545(293-879)$ & $<0.001$ \\
& Percent positive $(>301$ Units) & $53 \%$ & $74 \%$ & $<0.001$ \\
CLIFT & Titers (units) & $1: 160(1: 80-1: 320)$ & $1: 320(1: 160-1: 320)$ & $<0.001$ \\
& Percent positive $(>1: 10)$ & $99 \%$ & $97 \%$ & 0.26
\end{tabular}

Titers are presented as median inter-quartile range.

Table 2. Linear Mixed model effects of anti-dsDNA with ns-SELENA-SLEDAI scores

\begin{tabular}{lccc}
\hline Assay & Intercept + Estimate SE & $p$ value & Marginal $R^{2}$ \\
\hline QUANTA Flash & $3.3 \pm 0.5+0.0011 \pm 0.001$ & $<0.001$ & 0.112 \\
High Avidity & $2.8 \pm 0.8+0.0023 \pm 0.0011$ & 0.001 & 0.082 \\
QUANTA Lite & $2.9 \pm 0.6+0.0037 \pm 0.001$ & 0.022 & 0.037 \\
CLIFT & $3.3 \pm 0.8+0.0031 \pm 0.003$ & 0.28 & 0.006 \\
\hline
\end{tabular}

Conclusions: These preliminary data indicate that anti-dsDNA antibodies determined by QUANTA Flash have value in monitoring SLE disease activity.
Disclosure of Interest: T. Dervieux: None declared, T. O'Malley: None declared, J. Conklin: None declared, C. Ibarra: None declared, C. Bentow Employee of: Inova Diagnostics, M. A. Aure Employee of: Inova Diagnostics, M. Mahler Employee of: Inova Diagnostics

DOI: 10.1136/annrheumdis-2017-eular.5317

\section{SAT0663 TWO-STEP ALGORITHM FOR EARLY DIAGNOSIS OF OSTEOARTHRITIS BASED ON PLASMA DAMAGED AMINO ACIDS PROVIDES SIMPLE AND LOW COST SCREENING TEST}

N. Rabbani, U. Ahmed, P.J. Thornalley. Warwick Medical School, University of Warwick, Coventry, United Kingdom

Background: Currently, magnetic resonance imaging techniques have been developed for evaluation of cartilage damage in early-stage osteoarthritis (eOA). These imaging techniques have approximately $70 \%$ sensitivity and $90 \%$ specificity compared to reference diagnosis by arthroscopy. They require expensive instrumentation, time and facilities. In searching for biomarkers for clinical diagnosis we found that the proteolysis of damaged (oxidised, glycated and nitrated) proteins gives a unique pattern in the plasma of arthritic patients with the severity of the disease - trace level oxidised, glycated and nitrated amino acids. In this study we developed a two-step algorithm using these analytes as features.

Objectives: The objective was to distinguish between the following four groups: healthy control, eOA, early stage rheumatoid arthritis (eRA) and other inflammatory joint disease (non-RA).

Methods: Four algorithm types were tested for performance using random forests, multiclass logistic regression, multi-class sparse logistic regression and support vector machines. In all cases, the diagnostic algorithms were trained on the training data set, before being used to predict the disease class for each sample in the test data set. A two-stage approach was taken: (i) to distinguish between disease and healthy control; and (ii) to distinguish between eOA, eRA and non-RA. The area under the curve of the receiver operating characteristic plot (AUROC) statistic was used as measure of performance

Results: Random forest was the best-performing method. Application of two algorithms consecutively gave the best diagnostic outcome. The AUROC (sensitivity/specificity) values for disease/health were: eOA, $0.99(0.92 / 0.91)$; eRA, $0.96(0.89 / 0.90)$ and non-RA, $0.77(0.73 / 0.72)$ for the training set and test set validations. A random outcome is 0.50 . For typing arthritis, eOA, eRA and non-RA, AUROC values were in the range $0.68-0.98,0.77-0.93$ and $0.62-0.91$ for training set and test set cross-validations and test set validation, respectively. A random outcome is 0.33 .

Conclusions: A two-step algorithm approach based on trace level damaged amino acids gave better diagnostic performance that MRI for detecting and typing eOA. It is low cost and suitable for rollout as a clinical screening test.

References:

[1] Menashe, L., Hirko, K., Losina, E., Kloppenburg, M., Zhang, W., Li, L. and Hunter, D. J. (2012) The diagnostic performance of MRI in osteoarthritis: a systematic review and meta-analysis. Osteoarthritis and Cartilage. 20, 13-21. [2] Ahmed, U., Anwar, A., Savage, R.S., Thornalley, P.J. and Rabbani, N. (2016) Application of protein glycation, oxidation and nitration for early-stage diagnosis and severity of osteoarthritis and other arthritic disease. Arthritis Research \& Therapy 18:250.

Disclosure of Interest: None declared

DOI: 10.1136/annrheumdis-2017-eular.6904

\section{SAT0664 IS THERE AN EARLY ULTRASONOGRAPHIC PATTERN IN SALIVARY GLANDS IN BOTH PRIMARY AND SECONDARY SJOGREN SYNDROME?}

V.C. Iorgoveanu ${ }^{1}$, D. Mazilu ${ }^{2}$, I. Saulescu ${ }^{3}$, V. Vlad ${ }^{4}$, V. Bojinca ${ }^{3}$, L. Groseanu ${ }^{3}$, D. Opris ${ }^{3}$, A. Balanescu ${ }^{3}$, C. Constantinescu ${ }^{3}$, D. Predeteanu ${ }^{3}$, R. Ionescu ${ }^{3}$. ${ }^{1}$ Rheumatology, Carol Davila University of Medicine and Pharmacy; ${ }^{2}$ Saint Mary Clinical Hospital, UMF Carol Davila, Bucharest, Romania; ${ }^{3}$ Rheumatology, Saint Mary Clinical Hospital, UMF Carol Davila; ${ }^{4}$ Rheumatology, Saint Mary Clinical Hospital, Bucharest, Romania

Background: Sjogren Syndrome (SS) affects mainly exocrine glands. Ultrasonography (US) demonstrates specificity and sensibility in major salivary glands (SG) evaluation. Recent data confirm US might be used as primary evaluation technique for its ability to show structural alterations of parenchyma [1].

Objectives: To assess the gray scale (GS) parenchymal inhomogeneity of major SG in patients with established primary and secondary SS and correlate with clinical and biological data.

Methods: Consecutive patients with SS were recruited and SG US was performed. Inhomogeneity of glandular parenchyma was quantified binary on each gland. ESSDAI and ESSPRI scores were calculated. Statistics was performed with SPSS.

Results: Twenty one (42.85\% primary SS, $90.47 \%$ female) consecutive patients were included. Mean age was $53.66+/-12.99$ years and disease duration $5.33+/-$ 3.74 years. Antibody SSA/SSB presence was found in $85.7 \%(18 / 21)$. ESSDAI mean was $8.67+/-8.9(0-29)$, ESSPRI $10.13+/-5.59(0-20)$. There were no differences regarding ESSDAI and ESSPRI in the two groups (primary and secondary SS). Right parotid gland showed alterations in $71.4 \%$ patients $(77 \%$ 